\title{
Efficient and Effective Solid Waste Management in IoT-enabled Smart Cities
}

\author{
Kristian Alex Dame ${ }^{\mathrm{a}}$, Chi-Yuan Ha ${ }^{\mathrm{a}}$, Che-Wei Chang ${ }^{\mathrm{a}, \mathrm{b}, *}$ \\ ${ }^{a}$ Department of Computer Science and Information Engineering, School of Electrical and Computer Engineering, \\ College of Engineering, Chang Gung University, Tao-Yuan, 33302, Taiwan \\ ${ }^{\mathrm{b}}$ Department of Physical Medicine and Rehabilitation, Chang Gung Memorial Hospital, Tao-Yuan, Taiwan
}

*Corresponding Author: chewei@mail.cgu.edu.tw

\begin{abstract}
The concept of smart cities has been proposed to address critical problems in modern cities to improve the quality of social environment, economic environment, socio-cultural environment, medical/health systems, education, public services and transportation. One of the critical issues is to keep a city clean and tidy, and this work proposes an efficient and effective solid waste management system by the support of IoT infrastructure. With sensors in trash cans, the remaining capacity of each trash can be real-time monitored. The scheduling and routing algorithms are then developed to schedule the time points for sending out garbage trucks and to plan the route for each truck while the total cost of the solid waste management system is minimized. To evaluate our algorithms, a real case study was conducted with Manado City in Indonesia to prove the quality and practicability of our solid waste management system.
\end{abstract}

Keywords: smart cities, IoT, solid waste management.

\section{Introduction}

The concept of IoT (Internet of Things) has been proposed to make cities smart, more comfortable and able to give intelligent responses to different events or needs. Smart cities are then expected to act as driving force for improving the quality of city environment. One of the critical issues of the environment quality is on the solid waste management system for keeping a city clean and tidy. A fixed schedule of garbage trucks is a common solution for solid waste collection, but without the dynamic consideration of the garbage incoming rate of each trash can, the fixed schedule could be inefficient in terms of minimizing the management cost and keeping the environment clean. To address this problem, this work leverages the IoT infrastructure of a smart city, develops automatic garbage-truck scheduling and route planning algorithms, and implements a prototype for a real case study of Manado City.

Various techniques and services have been developed with smart cities for better government services, transport/traffic management, energy saving, health care, innovative urban agriculture, and waste management. For the development of smart cities, IoT plays a key role in bridging devices to computing units and exploring knowledge form the environment [1]. A survey [2] further studies existing smart things, such as smart fridges, smart washing machines, smart TVs, smart shoes, and smart phones, and smart things are quite popular on crowdfunding platforms, such as Kickstarter. By considering critical parameters in developing economy, a study [3] defines and characterizes the essentials of smart cities in the context of developing countries and analyzes the cost and constraints for converting cities to "smarty cities".

When solid waste management is considered in the development of smart cities, a case study [4] jointly considers source reduction, reuse, recycling, composting, landfilling, and waste combustion to minimize the total cost of the solid waste management. Quantitative results from the case study is also presented as the sensitivity analysis of the proposed framework. Base on the GIS (Geographic Information System) of a city, the work [5] then proposes an information system for maintaining the states of trash cans of all areas in the city. The information can be used to identify clean and dirty areas and can then be used for 
developing an efficient solid waste management system. Another research uses a Smart-M3 platform [6] and places sensors inside trash cans to monitor the fullness level of each trash can. According to the amount of solid waste in each area, the proposed algorithm then determines the number of trash cans distributed in each area and the number of trash trucks for each route. However, they consider only static routes. In our work, the practicability and advantage of dynamic route planning are further studied.

A genetic algorithm [7] is developed to find out the optimal routing for solid waste collection in cities. In their work, the target problem is modeled as the TSP (Traveling Salesman Problem), and static routes are then generated to go through all nodes with the minimum total distance. Another study [8] formulates the problem as a mixed integer program to minimize the solid waste collection and transportation cost. Anagnostopoulos et al. [9] have proposed an interesting solid waste collection system, in which garbage trucks might have different capacities. Low capacity trucks can go through streets and alleys to collect solid waste from trash cans, and high capacity trucks can be the mobile depots of low capacity trucks and move solid waste to a landfill site.

In this paper, solid waste collection of a city or a large national park is considered. Solid waste is deposited in trash cans, and garbage trucks should go through all trash cans to collect solid waste. To reduce the management cost, the total length of the routes of all trash trucks should be minimized. Moreover, the incoming rates of solid waste of all trash cans can be different and might vary from time to time. Thus, by considering the real-time information collected from the sensors in trash cans, this paper dynamically schedules garbage trucks and generates a route for each truck. By using the data of the solid waste management of the downtown Manado City, a real case study was conducted to evaluate the performance of the proposed management system.

The rest of the paper is structured as follows: Section 2 presents the system architecture and formally defines the target problem of this paper. Section 3 develops the algorithms for scheduling garbage trucks and planning routes. A real case study of Manado City is presented in Section 4, and Section 5 concludes this paper.

\section{System Architecture}

This work addresses the solid waste collection problem

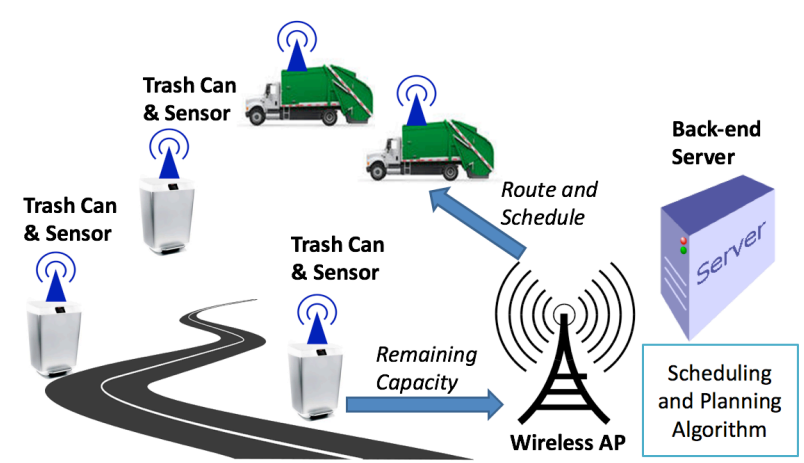

Fig. 1. System Architecture.

for a city or a national park and proposes a series of algorithms to reduce the total cost. The considered environment is illustrated in Fig. 1. In the considered environment, garbage is thrown into trash cans, and each trash can is equipped with a sensor to measure the remaining capacity of the trash can. Sensors in trash cans are then connected to Internet via $3 \mathrm{G} / 4 \mathrm{G}$ or wired network connection. The real-time data of the incoming rate of garbage and the remaining capacity of each trash can are then collected by a back-end server running our algorithms. The derived results of truck scheduling and route planning are then forwarded to garbage trucks. Garbage trucks follow the instructions from the back-end server to visit the designated trash cans by the given routes.

The target problem of this paper is to minimize the total cost of solid waste collection with the constraint that the environment (of a city or a park) has to be clean. For keeping the environment clean, whenever a trash can is full, a garbage truck has to be immediately sent out for collecting the solid waste in the trash can by the shortest route. However, some nearby trash cans might be going to be full soon and need to call another trash truck. Thus, it could be a good idea to modify the route to also go through the nearby trash cans which are going to be full soon. For the total cost of the solid waste collection, we define that in a period, the total cost of solid waste collection is the total length of the routes of the garbage trucks which have been sent out in the period. Therefore, the objective of this paper is to minimize the total length of routes in any period with the constraint that whenever a trash can is full, a garbage truck has to be sent out immediately for the garbage collection.

\section{Routing and Scheduling Algorithms}




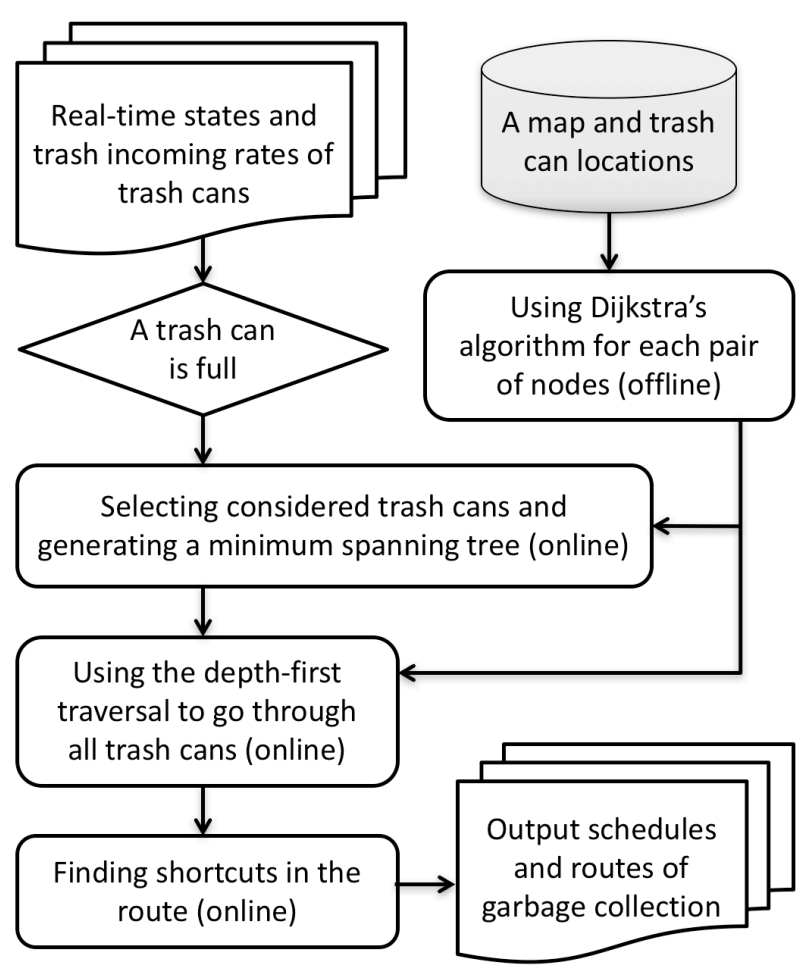

Fig. 2. Flow Diagram of the Solution.

Our solution consists of multiple algorithms to reduce the total length of routes without violating the constraint. A flow diagram is provided in Fig. 2 to summarize our solution. Trash cans and roads in a map are considered as nodes and edges in a graph and are analyzed to generate the shortest path for each pair of nodes. The real-time states and trash incoming rates of all nodes are considered as the input of the problem. When a node is full, other nodes which are going to be full are also picked up as considered nodes. A garbage truck should then go through all considered nodes and the full node and go back to the landfill site by using depth-first traversal and our shortcut algorithm. The solution details are in Sections 3.1 and 3.2.

\subsection{Input Analysis and Node Selection}

The input map of the trash cans and roads are analyzed to derive nodes and edges of a graph. Dijkstra's algorithm [10] is then used for the offline preprocessing to derive the shortest path of each pair of nodes in the graph. When the solid waste management system is online, if any node is full, a garbage truck should be sent out immediately. Before sending a garbage truck, we have to indicate the so-called considered nodes for the route of the to-be-sent garbage truck. Considered nodes are defined as the nodes which must be included in the route, and considered nodes are

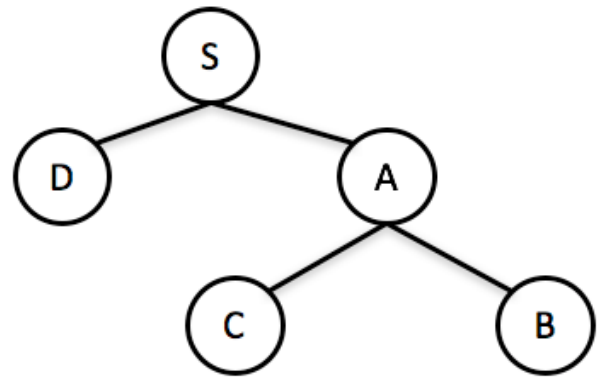

Fig. 3. A Minimum Spanning Tree.

selected by a garbage collection threshold. If the remaining capacity of a node is lower than the garbage collection threshold, the node is selected as a considered node. If the remaining capacity of a node is no less than the garbage collection threshold, the node is not selected as a considered node.

\subsection{Route Planning Algorithms}

After the considered nodes of a garbage truck are derived, our solution then has to derive a route to go through all of the considered nodes and the full node and make the length of the route as short as possible. To this end, a minimum spanning tree is constructed to connect all considered nodes, the full node, and the source node (the landfill site). With the minimum spanning tree, our solution then performs a depth-first traversal to start from the source node, go through all considered and full nodes, and go back to the source node. A minimum spanning tree is illustrated in Fig. 3 as an example, where $\mathrm{S}$ is the source node, and $\mathrm{A}$, $\mathrm{B}, \mathrm{C}$, and $\mathrm{D}$ are the considered nodes. The result of the depth-first traversal is $\mathrm{S} \rightarrow \mathrm{A} \rightarrow \mathrm{B} \rightarrow \mathrm{A} \rightarrow \mathrm{C} \rightarrow \mathrm{A} \rightarrow \mathrm{S} \rightarrow$ $\mathrm{D} \rightarrow \mathrm{S}$. However, it might be possible to build some shortcuts to reduce the length of this route. If the shortest path from $\mathrm{C}$ to $\mathrm{D}$ is shorter than the path $\mathrm{C} \rightarrow \mathrm{A} \rightarrow \mathrm{S} \rightarrow \mathrm{D}$, our solution will build the shortcut $\mathrm{C} \rightarrow \mathrm{D}$. If the shortest path from $\mathrm{B}$ to $\mathrm{C}$ is shorter than the path $\mathrm{B} \rightarrow \mathrm{A} \rightarrow \mathrm{C}$, our solution will build the shortcut $\mathrm{B} \rightarrow \mathrm{C}$. With the two shortcuts, the final route is $\mathrm{S} \rightarrow \mathrm{A} \rightarrow \mathrm{B} \rightarrow \mathrm{C} \rightarrow \mathrm{D} \rightarrow \mathrm{S}$.

\subsection{Property of the Solution}

Given a set of considered nodes, our route planning algorithm can achieve a 2-approximation bound of providing the shortest route for going through all considered nodes and the full nodes. For the considered problem, an algorithm is said to have a 2-approximation bound if the length of the provided route of the algorithm is always no more than twice the length of the optimal route. 
Since the minimum spanning tree [11] can connect all considered and full nodes with the source node with the minimum total length of the edges in the tree, the total length of the optimal route is always longer than that of the minimum spanning tree. Thus, the depth-first traversal of the minimum spanning tree is with the total length shorter than twice of the length of the optimal route. When some shortcuts are further used, the total length of the route of our solution only can be shorter. Thus, the 2-approximation bound of our solution is proven.

\section{Experiments with a Real Case Study}

This section presents the experimental setting and results of a real case study of Manado City. A file provided by the Manado City government shows that there are 30 large public trash cans in downtown Manado City, and the solid waste is collected by garbage trucks by fixed schedules in every week. There are 2 trash cans at the same location, and the other 28 trash cans are at different locations. The landfill site is considered as the source node in the graph. Therefore, there are are 30 nodes (including the source node) in our case study. The distance of each pair of nodes was generated by Google Map API and used as the length of the edge. In our experiments, five methods were implemented as follows:

Fixed-Schedule: The trash cans are partitioned into 7 groups by the Manado City government. When a node in a group is full, a garbage truck is sent to collect all the solid waste of the trash cans in the group.

Sol-10\%: It is our solution with the garbage collection threshold defined as $10 \%$.

Sol-20\%: It is our solution with the garbage collection threshold defined as $20 \%$.

Sol-30\%: It is our solution with the garbage collection threshold defined as $30 \%$.

Sol-40\%: It is our solution with the garbage collection threshold defined as $40 \%$.

The Fixed-Schedule method represents the traditional approach for solid waste collection, and it also has to meet the requirement that whenever a trash can is full, a garbage truck has to be sent out immediately.

The incoming rate of solid waste of each trash can was generated by a uniform distribution from 1 percent per hour (denoted as $\% / \mathrm{h}$ ) to $10 \% / \mathrm{h}$, where $\mathrm{X} \% / \mathrm{h}$ means that the incoming solid waste in one hour consumes $\mathrm{X} \%$ of the capacity of a trash can. When the remaining capacity of a trash can is $0 \%$, the trash can is considered as full, and a garbage truck is sent to collect the solid waste.

In the experiments, by considering the result of the Fixed-Schedule method as the baseline, all of the Sol-10\%, Sol-20\%, Sol-30\%, and Sol-40\% methods can reduce the total cost by more then $10 \%$. In some cases, the reduced cost can be more than $30 \%$. However, when we further studied the output routes of our solution, we found a problem in our current solution. When a node is full, our solution will pick up some other considered nodes. A garbage truck is then sent to go through the full node and the considered nodes with a given route. For selecting considered nodes, only the remaining capacities of nodes are examined, but our solution does not consider the locality of nodes. Thus, if there is a considered node very far from the full node, the cost of this route will be very high. In our feature work, the problem should be addressed to further improve the quality of our solution by weighting the nearby nodes of the full node for selecting the considered nodes. Nonetheless, our current solution still can guarantee that the reduced cost is always more than $10 \%$ compared with the Fixed-Schedule method in the case study of Manado City.

\section{Conclusion}

This work address the problem of solid waste collection to minimize the total cost of the system. Sensors in trash cans report the remaining capacities and the trash incoming rates, and our algorithms collects the data and generates schedules and routes. After the schedules and routes are provided, garbage trucks then go through their considered and full nodes with the given routes to collect solid waste. Analysis and a real case study were conducted to evaluate the performance of our solution. In our future work, large-scale simulations should be conducted to evaluate the scalability of the proposed solution. The spatial locality of nodes should be further analyzed to reduce the total cost. We believe this work can be practical and helpful for constructing a smart city.

\section{Acknowledgment}

The authors thank the Manado City government for providing the data. This work was supported by Chang Gung University and Chang Gung Hospital under grant No. NERPD2F0611 and grant No. BMRPD84, and by the Ministry of Science and Technology under grant No. MOST105-2221-E-182-030-MY2. 


\section{References}

(1) Andrea Zanella, Nicola Bui, Angelo Castellani, Lorenzo Vangelista, and Michele Zorzi : "Internet of things for smart cities", IEEE Internet Of Things, Vol. 1, No.1, pp. 22-32, 2014.

(2) Somayya Madakam : "Internet of things: Smart things", International Journal of Future Computer and Communication, Vol. 4, No. 4, pp. 250-253, 2015.

(3) Sheshadri Chatterjee and Arpan Kumar Kar : "Smart cities in developing economies: a literature review and policy insights", International Conference on Advances in Computing, Communications and Informatics, pp. 2335-2340, 2015.

(4) Stephen Chambal, Mark Shoviak, and Jr. Alfred E. Thal : "Decision analysis methodology to evaluate integrated solid waste management alternatives", Environmental Modeling and Assessment, Vol. 8, No. 1, pp. 25-34, 2003.

(5) Zhang Ruihong and Yu Zhichao : "The research of solid waste managemen system in dongguan city based on GIS", International Conference on E-Health Networking, Digital Ecosystems and Technologies, pp. 36-38, 2010.

(6) Vincenzo Catania and Daniela Ventura : "An approach for monitoring and smart planning of urban solid waste management using smart-m3 platform", Conference of Open Innovations Association FRUCT, pp. 25-31, 2014.

(7) Ingo von Poser and Adel R Awad : “Optimal routing for solid waste collection in cities by using real genetic algorithm", International Conference on Information and Communication Technologies, pp. 221-226, 2006.

(8) Swapan Das and Bidyut Kr.Bhattacharyya : "Optimization of municipal solid waste collection and transportation routes", International Journal of Integrated Waste Management, Science and Technology, Vol.43, pp. 9-18, 2015.

(9) Theodoros Anagnostopoulos, Arkady Zaslavsky, Stefanos Georgiou, and Sergei Khoruzhnicov : "High capacity trucks serving as mobile depots for waste collection in iot-enabled smart cities", International Conference on Internet of Things and Smart Spaces, pp. 80-94, 2015.

(10) Chuang Ruan, Jianping Luo, and $\mathrm{Yu} \mathrm{Wu} \mathrm{:} \mathrm{"Map}$ navigation system based on optimal Dijkstra algorithm", International Conference on Cloud Computing and Intelligence Systems, pp. 559-564,
2014.

(11) Ronald Lewis Graham and Pavol Hell : "On the history of the minimum spanning tree problem", IEEE Annals of the History of Computing, Vol. 7, No. 1, pp. 43-57, 1985. 